\title{
The effect of Cox-2 specific inhibition on direct fracture healing in the rabbit tibia
}

\author{
David J. Hak · Kurt S. Schulz • Behrang Khoie • \\ Scott J. Hazelwood
}

\begin{abstract}
Objective The purpose of this study was to evaluate the effect of Cox-2 administration on direct (primary) fracture healing.

Methods A transverse tibial osteotomy was created in adult male rabbits and rigidly fixed in compression using a 2.7-mm dynamic compression plate. Animals were randomized to receive either rofecoxib (12.5 mg orally per day) or placebo. Animals were killed at 4 weeks and fracture healing assessed by mechanical testing.

Results There were no significant differences between the control and Cox-2 treated animals in terms of mechanical strength at 4 weeks. There was a high complication rate of peri-implant fractures during the daily medication administration.

Conclusion The immediate administration of a Cox-2 specific inhibitor did not impair primary (direct) bone healing at the dose administered in this rabbit tibial osteotomy model.
\end{abstract}

\section{Introduction}

Fracture healing has classically been divided into either primary (direct) or secondary fracture healing. Primary fracture healing occurs when the cortex is anatomically reduced and rigidly fixed with a compression plate. The process involves osteoclasts in cutting cones crossing the fracture site and forming new osteons that bridge the fracture gap. In comparison, secondary bone healing occurs when there is relative motion between the fracture fragments and a large external callus is formed with subsequent endochondral and intramembranous bone formation.

The majority of preclinical studies have shown that nonspecific non-steroidal anti-inflammatory drugs (NSAIDs) impair secondary bone healing [1-4]. Several studies have also shown that a specific class of NSAIDs, cyclooxygenase-2 (Cox-2) specific inhibitors, delays healing and decreases fracture strength in animal models that heal by secondary bone healing [5-8]. These studies have examined the effect of Cox-2 inhibitors in a standard rat femoral fracture model in which the femur was loosely stabilized with a non-locked intramedullary k-wire. This method of fixation permits residual motion at the fracture site, and the fracture heals through the production of callus and secondary (indirect) bone healing. Other studies have shown that the administration of both nonspecific and specific Cox-2 inhibitors decreased the number of osteoclast-like cells in a bone ingrowth model [9]. Since osteoclasts play a critical role in primary bone healing, their impairment by Cox-2 inhibitors may conceivably lead to impaired primary (direct) bone healing.

Prior to the widespread publicity about increased cardiac complications associated with the use of certain Cox-2 inhibitors, Cox-2 inhibitors had become widely prescribed for common orthopedic conditions, and their use had been 
advocated as part of a multi-modality approach for postoperative pain control [10-15]. Following fracture fixation there is said to be a race between fracture healing and failure of fixation. If Cox-2 specific inhibitors impair primary (direct) bone healing, their use following rigid internal fixation of fractures might lead to fixation failure and nonunion. The purpose of this study was to investigate whether Cox-2 inhibitors impair primary (direct) bone healing in fractures that are anatomically reduced and rigidly fixed.

\section{Methods}

\section{Animal model}

One-year-old skeletally mature male New Zealand White rabbits were used under a protocol approved by our Institutional Animal Use and Care Committee. Animals ranged in weight from 3.0 to $3.5 \mathrm{~kg}$ with similar mean weights between the two groups. Anesthesia was initiated with a SQ injection of ketamine $(50 \mathrm{mg} / \mathrm{kg})$, xylazine $(5 \mathrm{mg} / \mathrm{kg})$ and AcePromazine $(0.5 \mathrm{mg} / \mathrm{kg})$, and maintained with alternating IV injections of ketamine $(20 \mathrm{mg})$ and xylazine $(2 \mathrm{mg})$ as needed. An intravenous catheter was inserted into the dorsal ear vein and $0.9 \%$ sodium chloride or LRS infused throughout the procedure $(10 \mathrm{mg} / \mathrm{kg} / \mathrm{min})$. The antibiotic Baytril $(10 \mathrm{mg} / \mathrm{kg}$ ) was administered IM preoperatively. Postoperatively the animals received Buprenorphine IM $(0.01 \mathrm{mg} / \mathrm{kg})$ and Baytril IM (10 mg/kg) every $12 \mathrm{~h}$ for 3 days.

The animals right hindlimb was prepped with betadine and sterilely draped. A longitudinal incision was made over the anterior tibial crest. A saline-cooled oscillating saw was used to produce a transverse mid-diaphyseal tibial osteotomy to simulate a fracture [16]. The osteotomy was anatomically reduced and internally fixed in compression with a six-hole, 2.7-mm dynamic compression plate (Synthes, Paoli, PA) and six bicortical screws. AP and lateral radiographs were obtained immediately postoperatively to document the reduction and fixation accuracy.

Animals were randomized to receive either placebo (sugar pill) or rofecoxib (Merck and Co., Inc., Whitehouse Station, NJ) $12.5 \mathrm{mg} /$ day administered orally once daily for 4 weeks and then discontinued. The tablet was covered in a tasty jelly and inserted directly into the rabbit's mouth by a veterinary technician.

The animals were housed individually in standard cages and provided fresh water and chow ad libitum. Animals were maintained for 28 days and then euthanized. Two additional animals that received placebo for the first 28 days were maintained for a total of 8 weeks in order to examine the osteotomy healing rate. Animals in which there was evidence of fixation loss or infection were excluded from the study and humanely euthanized.
Mechanical testing

After sacrifice, both tibia were harvested and cleaned of soft tissues. The plate was removed and the ends of the bone potted in methacrylate. The specimens were secured in a custom fixture for torsion testing and mounted in an Instron 8511 Universal Testing Machine (Instron Corp., Norwood, MA). Specimens were tested in torsion at a rate of $10^{\circ}$ per minute until failure. Rotational displacement and torque data were collected at $100 \mathrm{~Hz}$. Maximum torque was measured directly from the data, and torsional stiffness was calculated from a regression of the linear portion of the torque versus angular displacement curve. The energy absorption to failure was also calculated as the area under the curve to the maximum value. The nonfractured contralateral intact femur was also tested to calculate normalized values of maximum torque (percent maximum torque), stiffness (percent stiffness) and energy absorption to failure (percent energy) in order to reduce the influence of individual animal differences.

The location of the failure with respect to the osteotomy site was recorded. Specimens that failed directly through the osteotomy site were classified as nonunions. Specimens that failed with a spiral fracture that crosses the fracture site were classified as a union. Specimens that show some evidence of bone bridging at the osteotomy site but that failed primarily through the osteotomy were be classified as an incomplete union [17].

\section{Histology}

Following mechanical testing the specimens were removed from their potting fixture, and the two fragments of the fracture reapproximated and held with narrow gauge wire. The specimens were fixed in $4 \%$ paraformaldehyde with $0.1 \mathrm{M}$ phosphate buffer for $24 \mathrm{~h}$. They were then defatted in $70 \%$ ethanol and decalcified with $10 \%$ formic acid in citrate. The specimens were sliced in half longitudinally and embedded in paraffin. Paraffin sections ( $4 \mu \mathrm{m}$ thick) were cut and stained with hematoxylin and eosin.

\section{Statistical analysis}

A pre hoc power analysis was performed based on an average control maximum torque to failure of $1.8 \mathrm{~N} \mathrm{~m}$ with a standard deviation of 0.2 . Assuming a $20 \%$ difference, five animals per group were required to provide a $80 \%$ power with an alpha level of 0.5 . The means and standard deviations of the mechanical test data of osteotomized and intact tibia were calculated. The 95\% confidence interval for the mean was calculated for each group. An unpaired $T$ test with unequal variance was used to compare the mechanical results of the rofecoxib-treated animals with the placebo-treated animals. 
Table 1 Mechanical test results of 4-week animals (mean and standard deviation)

\begin{tabular}{lllll}
\hline Treatment & Side & $\begin{array}{l}\text { Maximum torque } \\
\text { to failure }(\mathrm{N} \mathrm{m})\end{array}$ & Stiffness $(\mathrm{N} \mathrm{m} / \mathrm{deg})$ & $\begin{array}{l}\text { Energy absorption to } \\
\text { maximum torque }(\mathrm{N} \text { m deg) }\end{array}$ \\
\hline Placebo $(n=5)$ & Osteotomy & $1.448+0.386$ & $0.127 \pm 0.059$ & $14.278 \pm 4.453$ \\
& Intact & $2.845 \pm 0.383$ & $0.153 \pm 0.026$ & $24.882 \pm 6.033$ \\
Rofecoxib $(n=7)$ & Osteotomy & $1.581 \pm 0.302$ & $0.117 \pm 0.057$ & $16.396+2.786$ \\
& Intact & $3.218 \pm 0.535$ & $0.163 \pm 0.022$ & $30.269+7.256$ \\
\hline
\end{tabular}

\section{Results}

There was a high rate of peri-implant fracture during animal handling for administration of the study medication in the immediate postoperative period. Sixteen animals sustained some peri-implant fracture or loss of fixation, and were euthanized prior to study completion. Fifteen of these failures occurred during the first 6 postoperative days, while one occurred at day 25. Loss of fixation and healing with angulation and callus occurred in four other animals (three placebo, one rofecoxib). This was discovered on radiographs and examination at the time of sacrifice, and these specimens were excluded from the study. Twelve animals, maintained for 4 weeks, whose radiographs showed stable fixation and no loss of alignment were used for mechanical analysis. Seven of these animals received rofecoxib, and five received placebo.

All specimens failed with production of a spiral fracture. None of the specimens failed solely through the osteotomy site. In the 4-week animals, seven specimens failed partially through the osteotomy site ( 3 placebo, 4 rofecoxib) and were categorized as incomplete unions. Five specimens ( 2 placebo, 3 rofecoxib) failed outside of the osteotomy site, usually though a distal screw hole, and were categorized as unions. In the 8-week animals, one failed partially through the osteotomy site (incomplete union), and one failed outside the osteotomy site (union).

At 4 weeks, the difference in the osteotomy site maximum torque to failure (Table 1) between the placebo- and the rofecoxib-treated animals was not significant $(p=$ 0.54 ). The $95 \%$ confidence interval of the osteotomy site maximum torque of the placebo group was $0.34-1.79 \mathrm{~N} \mathrm{~m}$ and of the rofecoxib group was $0.22-1.80 \mathrm{~N} \mathrm{~m}$. When normalized to the intact side, the $\%$ maximum torque to failure of the osteotomy site was approximately $50 \%$ of the intact side for both the placebo and rofecoxib groups (Fig. 1).

There was also no significant difference between the placebo- and rofecoxib-treated groups for stiffness $(p=0.77)$ or for the energy absorption to maximum torque $(p=0.38)$. The $95 \%$ confidence interval of the osteotomy site stiffness of the placebo group was $0.05-0.18 \mathrm{~N} \mathrm{~m} / \mathrm{deg}$ and of the rofecoxib group was $0.04-0.16 \mathrm{~N} \mathrm{~m} / \mathrm{deg}$. The

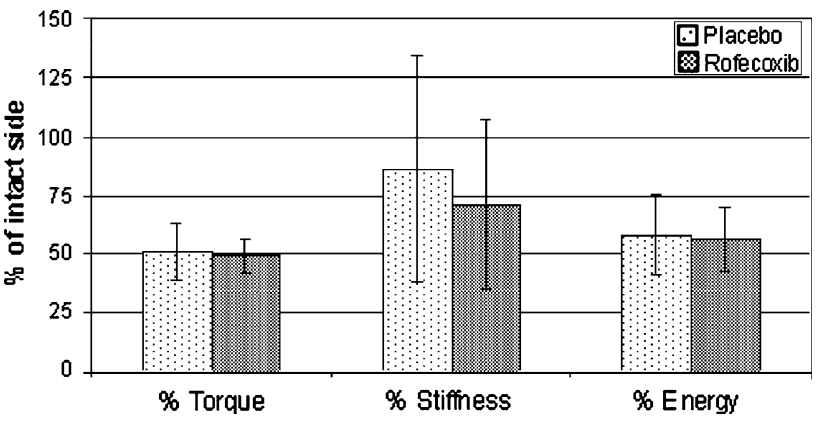

Fig. 1 Normalized values and standard deviations of mechanical test data: maximum torque, stiffness and energy absorption to maximum torque

95\% confidence interval of the osteotomy site energy absorption to maximum torque for the placebo group was $3.90-18.18 \mathrm{~N} \mathrm{~m} \mathrm{deg}$ and for the rofecoxib group was $2.06-18.46 \mathrm{~N} \mathrm{~m}$ deg. Normalized values for the stiffness came closer to approximating the intact side for both the placebo group and the rofecoxib group; however, the standard deviation was large.

The maximum torque to failure of the two animals treated with placebo and maintained for a total of 8 weeks was 1.50 and $1.68 \mathrm{~N} \mathrm{~m}$, which was not significantly different from that seen in the placebo group at 4 weeks (average maximum torque $1.45 \mathrm{~N} \mathrm{~m}$ ).

Intramembranous bone formation, aligned perpendicular to the longitudinal axis, was found to be present in the narrow gap present between the ends of the osteotomy sites (Fig. 2). Evidence of osteoclastic cutting cones with formation of new osteons was seen traversing the healed osteotomy sites (Fig. 3). Many of the specimens also showed some periosteal bone formation adjacent to the osteotomy site.

\section{Discussion}

In this rabbit tibial osteotomy model, we did not find any significant difference in the mechanical strength of direct fracture healing in animals treated with rofecoxib $(12.5 \mathrm{mg}$ daily dose) compared with placebo. However, we must caution that due to the small sample size we may not have been able to identify an actual difference between the 


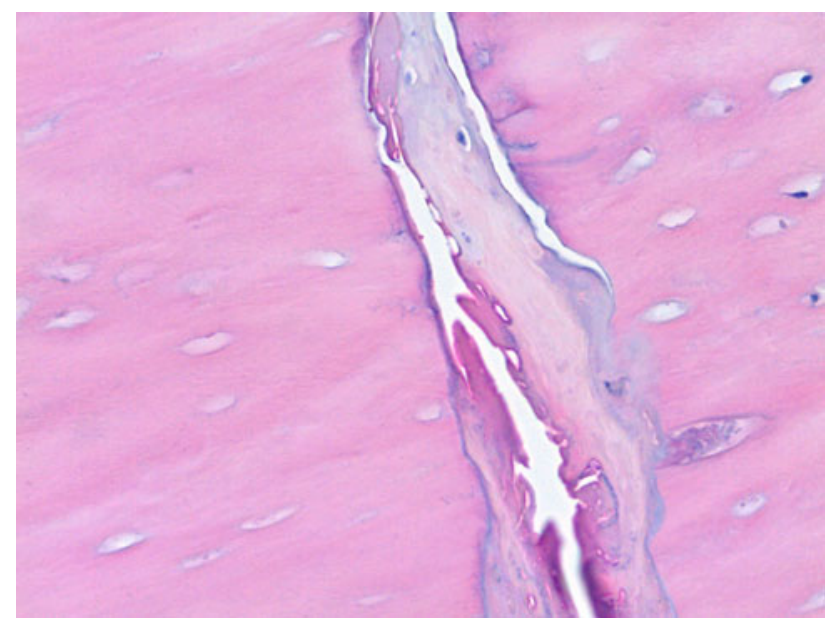

Fig. 2 Intramembranous bone formation is seen filling the narrow gap present between the ends of the osteotomy sites in a placebotreated animal. Note that the bone is aligned perpendicular to the longitudinal axis. $\mathrm{H} \& \mathrm{E}, \times 20$

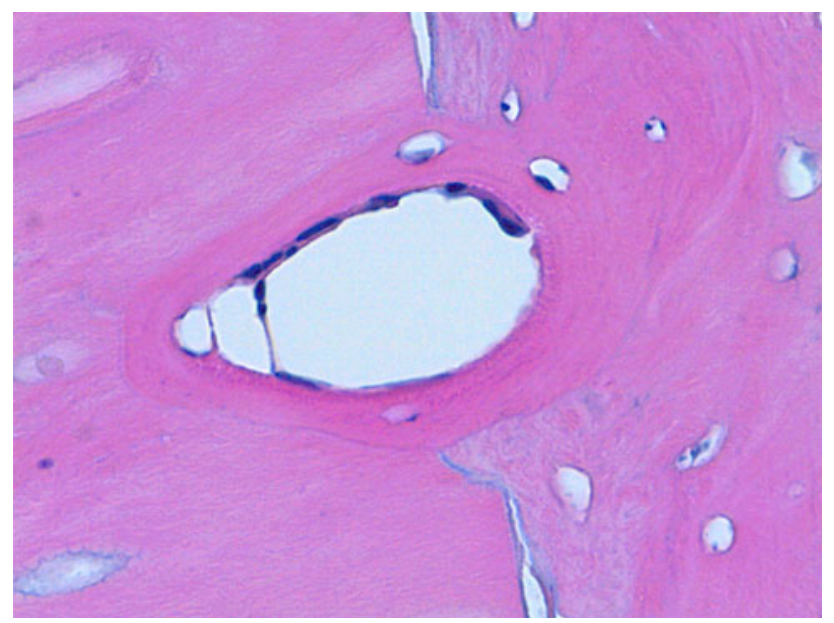

Fig. 3 New osteonal bone formation seen traversing the osteotomy site in a rofecoxib-treated animal. $\mathrm{H} \& \mathrm{E}, \times 40$

groups that may exist. The model selected for this study resulted in an unacceptably high complication rate, with a peri-implant fracture occurring in 16 animals and loss of fixation leading to fracture angulation in an additional 4 animals. The high complication rate with this animal model leads us to discourage its use for other studies examining primary (direct) bone healing.

Absolute stability with interfragmentary compression is necessary to achieve primary (direct) bone healing. We attempted to achieve interfragmentary compression of the transverse osteotomy site by eccentric screw placement in the holes of the $2.7 \mathrm{~mm}$ dynamic compression plates. The presence of a small gap between the ends of the osteotomy site in most sections suggests that only limited interfragmentary compression was achieved. In addition, the presence of periosteal bone formation suggests that there may have been some residual motion present at the osteotomy site. Alternatively this bone formation could be reactive due to inadvertent elevation of the periosteum around the osteotomy site. The presence of new osteonal bone formation traversing the osteotomy site does suggest that the model does involve primary (direct) bone healing. Because many of the healed specimens contained a combination of primary (direct) bone healing and periosteal bone formation adjacent to the osteotomy site, our mechanical test data do not reflect the contribution of primary (direct) bone healing alone.

As with any study there were problems and weaknesses associated with our investigation. The rofecoxib dosage selected was based on that used in prior studies; however, this may not be the appropriate effective dose due to differences in absorption and metabolism in this species [9]. We chose to give the medication directly into the rabbit's mouth, rather than in their feed, to ensure consistent administration. Many fractures occurred because of the rabbits' forced hindlimb movements during medication administration. Even in animals in which catastrophic failure did not occur, loss of fixation still occurred, leading to further exclusion of animals. Because of the difficulties using this model we did not have separate specimens available for a more thorough quantitative histologic evaluation.

We examined only a single 4-week timepoint and may have missed subtle differences that rofecoxib may have had on the rate of fracture healing. We chose to examine the mechanical strength of healing at 4 weeks; however, other investigators have shown that the strength of plated rabbit tibia increased up to 6 weeks when tested in bending mode [18]. Because the process of primary bone healing is more gradual than secondary bone healing, the 4-week timepoint may have been too early since some other investigators have reported that osteonal bridging occurred at 6 weeks or later [16]. Difficulties with the animal model led us to abandon plans to study a larger group of animals at an 8-week timepoint.

There is conflicting information about the effect of Cox2 inhibition on fracture healing. Several pre-clinical studies have reported that Cox-2 inhibitors result in impaired fracture healing in animal models that heal by secondary (indirect) bone healing [5-8]. The magnitude of the detrimental effect of selective Cox-2 inhibitors on secondary fracture healing has been shown to be related to the duration of treatment [6]. Celecoxib administered during the first 2 weeks after fracture significantly impaired fracture healing in a rat secondary fracture healing model, whereas treatment prior to fracture or initiated 14 days after fracture did not impair secondary fracture healing [7].

Other studies, however, have reported that some Cox-2 inhibitors have a less detrimental effects on fracture 
healing than non-specific nonsteroidal anti-inflammatories [19-22]. Brown and colleagues [19] found no decrease in the mechanical strength of rat femur fracture healing at 4,8 and 12 weeks in animals treated with celecoxib $(3 \mathrm{mg} / \mathrm{kg})$. While they did report that the celecoxib treated fractures appeared to have more fibrous tissue than controls at 4 and 8 weeks, they found no significant difference in the degree of radiographic callus and the mechanical strength. Simon and colleagues reported that rofecoxib ( $4 \mathrm{mg} / \mathrm{kg})$ significantly diminished the mechanical properties of a standard rat femur fracture at 8 weeks, but that celecoxib $(3 \mathrm{mg} / \mathrm{kg})$ showed no significant difference in the mechanical properties of the fracture when compared to controls [20].

Karachalios et al. compared the effect of short-term administration of rofecoxib with non-selective NSAIDs and prednisolone on secondary fracture healing in a rabbit ulna osteotomy model. They administered rofecoxib $(0.5 \mathrm{mg} / \mathrm{kg} / 24 \mathrm{~h})$ orally for 5 consecutive days. While all the biomechanical parameters of prednisolone, indomethicin and meloxicam were significantly lower than in the control group, only the fracture load values of rofecoxib were lower than the control. The histomorphometric parameters showed the least negative effects in the rofecoxib group. The investigators concluded that the short-term administration of rofecoxib had only a minor negative effect on bone healing [21].

Gerstenfeld and colleagues [23] characterized the expression of Cox -1 and Cox -2 mRNA in rat fracture calluses at timed intervals after fracture using real-time PCR. They found that Cox-2 mRNA levels showed peak expression during the first 14 days of healing and returned to baseline levels by day 21 . In addition they examined the effect of ketorolac ( $4 \mathrm{mg} / \mathrm{kg}$ ), a non-specific NSAID, and two doses of parecoxib $(0.3$ and $1.5 \mathrm{mg} / \mathrm{kg})$, a pro-drug of valdecoxib, in a rat femur fracture model. They found significant reduction in the mechanical properties of animals treated with ketorolac at 21 days post-fracture. While the mean mechanical strength of the parecoxib-treated animals was less at 21 days, it was not statistically significant. They concluded that a Cox-2-selective NSAID such as parecoxib has only a small effect on delaying fracture healing, even at doses that are known to fully inhibit prostaglandin production.

Utilizing a knockout mouse model, Zhang and colleagues [24] demonstrated that Cox-2 plays an essential role in both endochondral and intramembranous bone formation during skeletal repair. The healing of stabilized tibia fractures was significantly delayed in the Cox-2-/mice compared to the Cox-1-/- and the control wild-type mouse. The histology of the fibrous nonunions in the Cox$2-/-$ mice was characterized by a persistence of undifferentiated mesenchyme and a marked reduction in osteogenesis.
While some authors have expressed caution in the use of Cox-2 inhibitors in patients following fracture or other surgery requiring bone healing, others have questioned the clinical importance of the published animal data [25]. Similar concerns have been raised about the larger body of literature that has examined the effect of non-Cox-2 specific nonsteroidal anti-inflammatories on fracture healing in animal models. However, two clinical reports have raised concern about the effect of NSAIDs on fracture healing. In one study, patients who received indomethicin as prophylaxis for heterotopic ossification following surgical treatment of acetabular and long bone fractures had a significantly greater rate of long bone nonunion (26 vs. $7 \%$, $p=0.004$ ) [26]. In a second retrospective study investigators found a statistically significant $(p=0.000001)$ association between nonunion of the femoral diaphysis and use of NSAIDs after injury [27].

While there may be other reasons to avoid the use of Cox-2 inhibitors in certain patients, the necessity for primary (direct) bone healing need not be a contraindication for their use. The immediate administration of a Cox-2specific inhibitor did not impair primary (direct) bone healing at the dose administered in this rabbit tibial osteotomy model.

Acknowledgments This work was supported by a grant from the Orthopaedic Trauma Association and a faculty research grant from the University of California, Davis.

Conflict of interest The authors have no conflict of interest related to the content of this study.

\section{References}

1. Allen HL, Wase A, Bear WT. Indomethicin and aspirin: effect of nonsteroidal anti-inflammatory agents on the rate of fracture repair in the rat. Acta Orthop Scand. 1980;51:595-600.

2. Altman RD, Latta LL, Keer R, Renfree K, Hornicek FJ, Banovac K. Effect of nonsteroidal anti-inflammatory drugs on fracture healing: a laboratory study in rats. J Orthop Trauma. 1995;9: $392-400$.

3. Ho ML, Chang JK, Wang GJ. Antiinflammatory drug effects on bone repair and remodeling in rabbits. Clin Orthop. 1995;313: $270-8$.

4. Høgevold HE, Grøgaard B, Reikerås O. Effects of short-term treatment with corticosteroids and indomethacin on bone healing: a mechanical study of osteotomies in rats. Acta Orthop Scand. 1992;63:607-11.

5. Bergenstock M, Min W, Simon AM, Sabatino C, O'Connor JP. A comparison between the effects of acetaminophen and celecoxib on bone fracture healing in rats. J Orthop Trauma. 2005;19: $717-23$.

6. Gerstenfeld LC, Al-Ghawas M, Alkhiary YM, Cullinane DM, Krall EA, Webb EG, Thiede MA, Einhorn TA. Selective and nonselective cyclooxygenase- 2 inhibitors and experimental fracture-healing. Reversibility of effects after short-term treatment. J Bone Joint Surg Am. 2007;89:114-25. 
7. Simon AM, O'Connor JP. Dose and time-dependent effects of cyclooxygenase-2 inhibition on fracture-healing. J Bone Joint Surg Am. 2007;89:500-11.

8. Herbendick MA, Sprott D, Stills H, Lawless M. Effects of a cyclooxygenase 2 inhibitor on fracture healing in a rat model. Am J Orthop. 2008;37:E133-7.

9. Goodman S, Ma T, Trindade M, Ikenoue T, Matsuura I, Wong N, Fox N, Genovese M, Regula D, Smith RL. Cox-2 selective NSAID decreases bone ingrowth in vivo. J Orthop Res. 2002;20:1164-9.

10. Bresalier RS, Sandler RS, Quan H, Bolognese JA, Oxenius B, Horgan K, Lines C, Riddell R, Morton D, Lanas A, Konstam MA, Baron JA. Adenomatous polyp prevention on Vioxx (APPROVe) trial investigators cardiovascular events associated with rofecoxib in a colorectal adenoma chemoprevention trial. $\mathrm{N}$ Engl J Med. 2005;352:1092-102.

11. Nussmeier NA, Whelton AA, Brown MT, Langford RM, Hoeft A, Parlow JL, Boyce SW, Verburg KM. Complications of the COX-2 inhibitors parecoxib and valdecoxib after cardiac surgery. New Engl J Med. 2005;352:1081-91.

12. Reuben SS, Fingeroth R, Krushell R, Maciolek H. Evaluation of the safety of the perioperative administration of rofecoxib for total knee arthroplasty. J Arthroplasty. 2002;17:26-31.

13. Ruoff G, Lema M. Strategies in pain management: new and potential indications for Cox-2 specific inhibitors. J Pain Symptom Manage. 2003;25(2):S21-31.

14. Sinatra R. Role of Cox-2 inhibitors in the evolution of acute pain management. J Pain Symptom Manage. 2002;24(1):S18-27.

15. Sinatra R, Torres J, Bustos AM. Pain management after major orthopaedic surgery: current strategies and new concepts. J Am Acad Orthop Surg. 2002;10:117-29.

16. Rahn BA, Gallinaro P, Baltensperger A, Perren SM. Primary bone healing: an experimental study in the rabbit. J Bone Joint Surg Am. 1971;53:783-6.

17. White A, Panjabi M, Southwick W. The four biomechanical stages of fracture repair. J Bone Joint Surg Am. 1977;59:188-92.
18. Terjesen T. Bone healing after metal plate fixation and external fixation of osteotomized rabbit tibia. Acta Orthop Scand. 1984;55:69-77.

19. Brown KM, Saunders MM, Kirsch T, Donahue HJ, Reid JS. Effect of COX-2-specific inhibition on fracture-healing in the rat femur. J Bone Joint Surg Am. 2004;86:116-23.

20. Simon AM, Manigrasso MB, O’Connor JP. Cyclo-oxygenase 2 function is essential for bone fracture healing. J Bone Miner Res. 2002;17:963-76.

21. Karachalios T, Boursinos L, Poultsides L, Khaldi L, Malizos KN. The effect of short-term administration of low therapeutic doses of anti-cox-2 agents on the healing of fractures: an experimental study in rabbits. J Bone Joint Surg Br. 2007;89:1253-60.

22. Mullis BH, Copland ST, Weinhold PS, Miclau T, Lester GE, Bos GD. Effect of cox-2 inhibitors and non-steroidal anti-inflammatory drugs on a mouse fracture model. Injury, Int J Care Injured. 2006;37:827-37.

23. Gerstenfeld LC, Thiede M, Seibert K, Mielke C, Phippard D, Svagr B, Cullinane D, Einhorn TA. Differential inhibition of fracture healing by non-selective and cyclooxygenase-2 selective non-steroidal anti-inflammatory drugs. J Orthop Res. 2003;21: $670-5$.

24. Zhang X, Schwarz EM, Young DA, Puzas JE, Rosier RN, O'Keefe RJ. Cyclooxygenase-2 regulated mesenchymal cell differentiation into the osteoblast lineage and is critically involved in bone repair. J Clin Invest. 2002;109:1405-25.

25. Hochberg MC, Melin JM, Reicin A. Cox-2 inhibitors and fracture healing: an argument against such an effect. J Bone Miner Res. 2003; 18:583.

26. Burd TA, Hughes MS, Anglen JO. Heterotopic ossification prophylaxis with indomethicin increases the risk of long-bone nonunion. J Bone Joint Surg Br. 2003;85:700-5.

27. Giannoudis PV, MacDonald DA, Matthews SJ, Smith RM, Furlong AJ, De Boer P. Nonunion of the femoral diaphysis: the influence of reaming and non-steroidal anti-inflammatory drugs. J Bone Joint Surg Br. 2000;82:655-8. 